\title{
Helical TomoTherapy for locally advanced or recurrent breast cancer
}

\author{
M. N. Duma ${ }^{1,3^{*}}$, C. Heinrich ${ }^{1,2}$, C. Schönknecht ${ }^{1}$, B. Chizzali' ${ }^{1}$ M. Mayinger ${ }^{1}$, M. Devecka' ${ }^{1}$ S. Kampfer ${ }^{1}$ \\ and S. E. Combs ${ }^{1,3}$
}

\begin{abstract}
Purpose: We report our experience of using helical tomotherapy $(\mathrm{HT})$ to treat large and irregular shaped locoregional advanced breast cancer target volumes embracing various organs at risk.

Patients and methods: We retrospectively analyzed 26 patients treated for very large, irregular shaped breast cancers. Patients were treated either with the intent to achieve local control in a primary setting $(n=14)$ or in a reirradiation setting $(n=12)$. The recurrence group was heavily pretreated with systemic therapy. Tumors were characterized by wide infiltration of the skin, encompassing mostly a complete hemithorax. The primary group underwent irradiation of supraclavicular, infraclavicular, axillary and parasternal lymphonodal region. Radiotherapy was combined with chemotherapy $(n=11)$. We assessed the PTV volume and its craniocaudal extension, the dose to the organs at risk, acute toxicity and survival.

Results: Median PTV was $2276 \mathrm{~cm}^{3}\left(1476-6837 \mathrm{~cm}^{3}\right)$ with a median cranio-caudal extension of $28 \mathrm{~cm}(15-52 \mathrm{~cm})$. The median dose to PTV was 40 Gy (32-60Gy). HT could be carried out in all patients without interruption. The acute toxicities were mild to moderate. The median LRFS and OS after radiotherapy was 21 and 57 months for the primary group versus 10 and 11 months for the recurrence group. Median PFS was 18 months (primary group) and 7 months (recurrence group).

Conclusions: HT is feasible for advanced thorax embracing target volumes with acceptable acute toxicity. Both curative and palliative indications can be considered good indications based on treatment volume and anatomical constellation.
\end{abstract}

Keywords: Helical tomo therapy, Breast cancer, Reirradiation, Chemoradiation, Acute toxicity

\section{Introduction}

The standard of care for the treatment of local breast cancer recurrence is surgery. High local control rates can be achieved with mastectomy after primary breast conserving therapy [1].

In case of inoperable locally advanced chest wall recurrences the standard treatment is radiotherapy [2]. However in most cases whole breast irradiation after breast conserving surgery was performed beforehand. Thus, exposure of

\footnotetext{
* Correspondence: Marciana.Duma@mri.tum.de

${ }^{1}$ Department of Radiation Oncology, Klinikum rechts der Isar der

Technischen Universität München, Ismaninger Str. 22, 81675 München,

Germany

${ }^{3}$ Institute of Innovative Radiotherapy (iRT), Helmholtz Zentrum München,

München, Germany

Full list of author information is available at the end of the article
}

preirradiated normal tissue (skin, soft tissues, bones, lungs, heart) limits the reirradiation options.

Radiotherapy for large and complexly shaped tumor volumes in case of local recurrence or primary locally advanced tumor is often not reasonably achievable with conventional 3D conformal radiotherapy (3D-CRT). This is mostly due to the fact, that the tumor partly embraces organs at risk (OAR) such as the lungs or heart [3]. Planning studies for the use of helical tomotherapy (HT) for breast cancer treatment have been shown to improve conformality to the tumor bed, while sparing organs at risk (OARs). While HT has been studied after breast conserving surgery in the context of partial breast radiation $[4,5]$, whole breast radiation [6] and loco regional nodal radiation [3] only few data are available on the feasibility, acute side effects and outcome of helical 
tomotherapy in the context of large chest wall recurrences and reirradiation and primary locally advanced tumor volumes $[7,8]$.

The following retrospective analysis presents a single university center experience with treatment of advanced breast cancer with/without chemotherapy using HT.

\section{Material and methods}

\section{Patient characteristics}

From April 2007 to August 2011, 26 patients (age range: 34-79 years) were treated for very large, irregular shaped local recurrences $(n=12)$ or for locally advanced breast cancer in a primary setting $(n=14)$ with HT. Table 1 depicts the patients' characteristics. HT was at the given time the only machine available in our department that could perform IMRT and (daily) CT based IGRT and treat extensive volumes.

\section{Reccurence group}

All patients in the recurrence group were treated with a palliative intent. Tumors in this group were characterized by wide infiltration of the skin and subcutaneous tissue as well as extensive lymph node metastases. Palliative treatment was offered for ulcerating tumors, pain and/or neurological symptoms in patients with extensive bulky disease. Six patients received an additional individualized boost treatment with neutrons. Neutrons are used on a routine basis in our department in a third or higher reirradiation setting of extended cutaneous/subcutaneous metastasis. Ten patients had distant metastases and ongoing systemic therapies. Most patients were heavily pretreated and systemic therapies varied depending on the pretreatment. For nine patients it was necessary to continue dose-reduced chemotherapy during reiradiation because of systemic progression. Nine of the 12 patients $(75 \%)$ in the recurrence group had initially received breast conserving surgery followed by whole breast radiotherapy (WBRT). Three patients had radical mastectomy. Figure 1 depicts a typical patient from the recurrence group.

\section{Primary setting group}

Patients in primary setting had locally advanced tumors with extensive involvement of axillary lymph nodes (bulky disease) and macroscopic parasternal lymph nodes or were at high risk of parasternal lymph nodes metastases. One patient underwent polychemotherapy without surgery. 8 of 14 (57\%) patients underwent

Table 1 Patients' characteristics

\begin{tabular}{|c|c|c|c|}
\hline & All patients & Recurrence Treatment Group & Primary Treatment Group \\
\hline \multicolumn{4}{|l|}{ Descriptive characteristics } \\
\hline$n$ & $26(100 \%)$ & $12(100 \%)$ & $14(100 \%)$ \\
\hline Median age (range) & $63(31-75)$ & $52(31-75)$ & $69(62-74)$ \\
\hline Median Karnofsky Performance Score (range) & $90 \%(70-90 \%)$ & $90 \%(70-90 \%)$ & $90 \%(80-90 \%)$ \\
\hline Distant metastases & $13(50 \%)$ & $6(50 \%)$ & $7(50 \%)$ \\
\hline \multicolumn{4}{|l|}{ Histology } \\
\hline Invasiv ductal & $21(81 \%)$ & $9(75 \%)$ & $12(86 \%)$ \\
\hline Invasiv lobular & $4(15 \%)$ & $2(17 \%)$ & $2(14 \%)$ \\
\hline Angiosarcoma & $1(4 \%)$ & $1(8 \%)$ & - \\
\hline \multicolumn{4}{|l|}{ Side } \\
\hline Left side & $12(46 \%)$ & $6(50 \%)$ & $6(43 \%)$ \\
\hline Right side & $10(38 \%)$ & $4(33 \%)$ & $6(43 \%)$ \\
\hline Bilateral & $4(15 \%)$ & $2(17 \%)$ & $2(14 \%)$ \\
\hline \multicolumn{4}{|l|}{ Symptoms at radiotherapy start } \\
\hline Exulcerating/painful tumour & $8(31 \%)$ & $6(50 \%)$ & $2(14 \%)$ \\
\hline Extensive lymph node metastases with lymphedema & $9(35 \%)$ & $5(42 \%)$ & $4(29 \%)$ \\
\hline \multicolumn{4}{|l|}{ Previous treatments } \\
\hline Breast conserving surgery & $15(58 \%)$ & $9(75 \%)$ & $6(43 \%)$ \\
\hline Median Dose WBRT & - & 60 Gy (50.4-66 Gy) & - \\
\hline Prior radical mastectomy & $14(54 \%)$ & $3(25 \%)$ & $11(79 \%)$ \\
\hline Prior polychemotherapy & $10(38 \%)$ & $6(50 \%)$ & $4(29 \%)$ \\
\hline Prior adjuvant endocrine treatment & $15(57 \%)$ & $4(33 \%)$ & $11(79 \%)$ \\
\hline
\end{tabular}

Depicted are absolute values or median values with range 

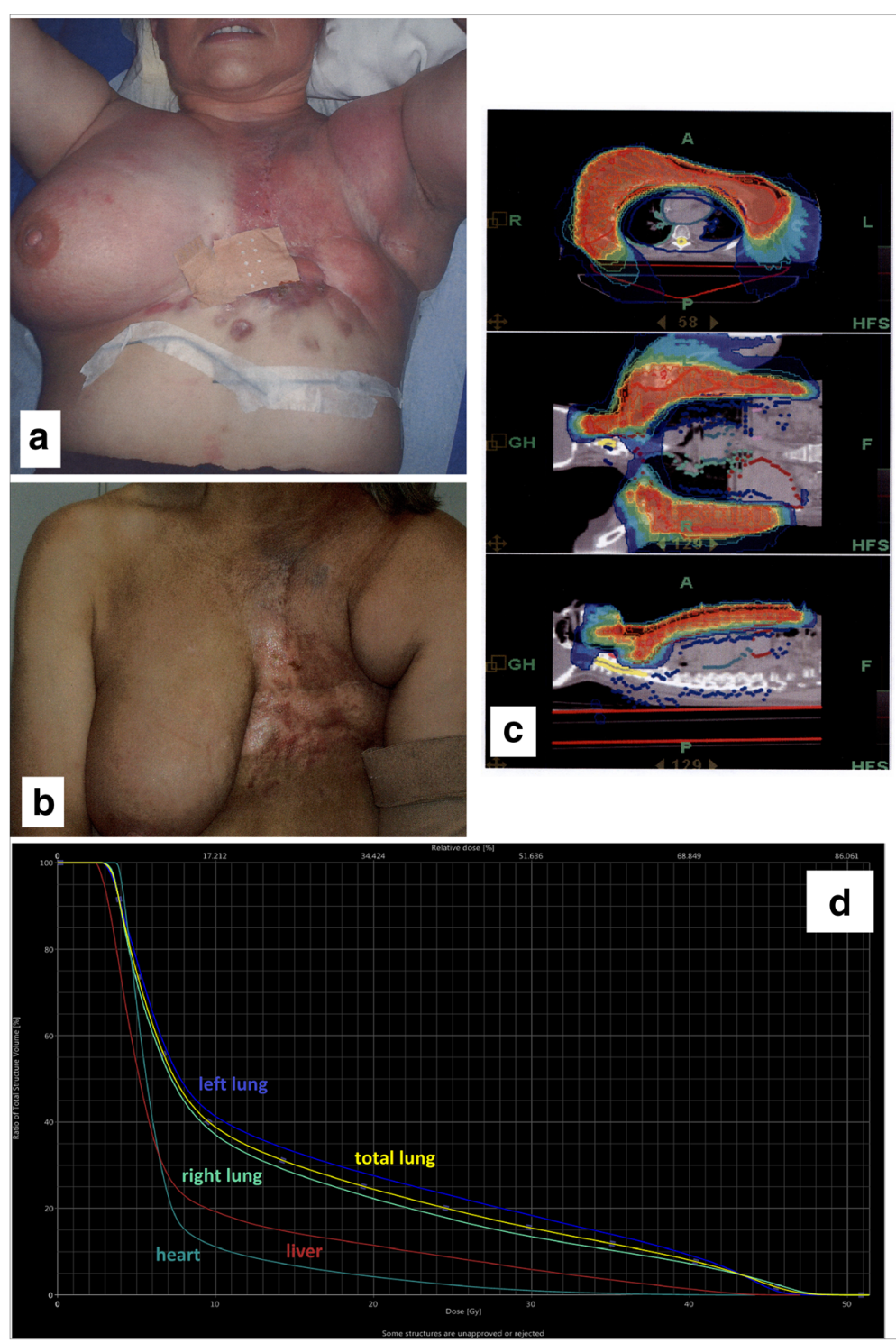

Fig. 1 Patient from the recurrence treatment group a: clinical appearence at the start of HT; $\mathbf{b}$ : clinical appearence 6 weeks after HT; c: HT treatment plan; $\mathbf{d}$ DVH

radical mastectomy and the remaining $6(43 \%)$ patients received breast conserving surgery. Nonetheless in 3 of these 6 patients radical mastectomy was necessary after a prior breast conserving attempt. Seven of the $14(50 \%)$ patients had positive resection margins (5 pts.- R1 resection, 2 pts.- R2 resection) after radical mastectomy. Five of the $11(45 \%)$ patients after radical mastectomy had extensive lymphangiosis carcinomatosa.

\section{Treatment planning and delivery}

Before treatment, each patient underwent a planning computer tomography (CT) scan (Siemens Somatom; Siemens Inc., Erlangen, Germany) with an axial slice thickness of $3 \mathrm{~mm}$. A vaccum bag (Bodyfix, Medical
Intelligence, Schwabmuenchen, Germany) was used for immobilization. The contouring of the PTV and the OARs was performed on the Oncentra Master-Plan system (Nucletron B.V., Veenendaal, The Netherlands) and iPlan (Brainlab, Feldkirchen, Germany). Treatment planning was done using Tomotherapy HI-Art System ${ }^{\circ}$ (Accuray Inc., Sunnyvale CA, USA). The prescribed dose to the PTV was dependent on previous irradiation regimes and ranged from 32 to 60 Gy with a median PTV dose of $40 \mathrm{~Gy}$. Organs at risk (OARs) were contoured according to internal guidelines and constraints were set according to Emami et al. [9]. In case of reirradiation, the spinal cord biological equivalent dose was calculated according to Nieder et al. [10]. All patients underwent 
daily image guided radiotherapy (IGRT). After acquisition and reconstruction, the daily megavoltage CT scans (MVCT) were automatically registered, by choosing the bone and tissue algorithm provided by TomoTherapy, to the planning CT. To account for the best positioning of the patient, every automatic registration was controlled and corrected by experienced staff members before treatment.

\section{Outcome}

Acute toxicity was assessed using the Radiation Therapy Oncology Group/National Cancer Institute Common Toxicity Criteria (RTOG/NCI CTC), version 3.0, morbidity scales. Acute toxicity was assessed every week during HT and 6-8 weeks after treatment. The planned follow up visits were: $6-8$ weeks after HT, 3, 6 and 12 months after HT and thereafter once a year. Most clinical follow up data presented herein on local control was collected from the first visit 6-8 weeks after treatment. Many patients were then lost to follow up and there is not enough data available to reliably assess the late side effects. Treatment response was measured by clinical examination and radiological findings. Complete response was defined as the clinical disappearance of all irradiated target lesions. A partial response was defined as a decrease of more than $50 \%$ of the target lesion. Stable disease was defined as a decrease less than $50 \%$ or no change. Progressive disease was defined as an increase of target lesions or appearance of new lesions ("in field" or throughout the body). Data for PFS, LRFS and OS was collected from different sources (patient records, data from primary oncologists, the Munich Cancer Registry). Data are reported by means of descriptive statistics and Kaplan Meier survival function.

\section{Results}

\section{Treatment parameters}

HT could be carried out to the planned cumulative dose in all patients without interruption.

Table 2 depicts the treatment parameters for both treatment groups.

12 patients with extensive local recurrences received a total reirradiation dose which ranged from 32 to 60 Gy total dose (1.8-3 Gy daily) depending on palliative need, time between initial therapy and retreatment and initial irradiation dose. Two patients were treated with a simultaneous integrated boost, one patient with electron boost and six with neutron boost. The median interval between initial radiotherapy and reirradiation was 2.4 years (1-21 years). The median dose from the previous radiotherapy was $60 \mathrm{~Gy}$ (50.4-66 Gy). The 14 patients with primary locally advanced tumors received a total dose between 40 and $60 \mathrm{~Gy}$. Three patients were treated with a simultaneous integrated boost.
The median mean dose (Dmean) to the heart in the primary and retreatment group was 13 Gy (1.5-34 Gy) and 12Gy (6-22 Gy) and the median Dmean to the lung was $21 \mathrm{~Gy}(12-25.5 \mathrm{~Gy})$ in the primary treatment group and 16Gy (5-20 Gy) in the retreatment group, respectively. Overall, the V5 was 95\% (25-100\%); the V20 18\% (5-45\%) and the V30 12\%(1-27\%). Overall 11 patients received a combined radiochemotherapy depending on pretreatment, systemic progression and kidney function (Table 2). Five patients received capecitabine $(825 \mathrm{mg} /$ $\mathrm{m}^{2}$ bid, including weekends), Four patients vinorelbine (25 mg/m ${ }^{2} 8 \mathrm{q}$ or $\left.60 \mathrm{mg} / \mathrm{m}^{2} 14 \mathrm{q}\right)$, one patient doxorubicin $(40 \mathrm{mg}$ q28d) and one patient sunitinib $(12,5 \mathrm{mg}$ daily). Concurrent chemotherapy was well tolerated and could be carried out as planned during HT treatment without interruptions. Additionally, four patients continued endocrine treatment (two patients anastrozol, two patients letrozol).

\section{Acute side effects}

The acute toxicity was mild to moderate measured weekly during therapy, 6-8 weeks and three to 4 months after treatment. Table 3 depicts the acute side effects.

\section{Outcome}

Overall, at the end of the treatment $23 \%(n=6 / 26)$ patients had a local partial remission, 69\% $(n=18 / 26)$ a stable disease, $7.7 \%(n=2 / 26)$ a local progressive disease and $15 \%(n=4 / 26)$ had already a systemic progression. Six to eight weeks after HT 50\% $(n=6 / 12)$ of the retreatment group showed a local partial remission and 33\% (4/12) showed a complete local remission "in field". All four patients received a neutron boost.

Table 4 depicts the local outcome and the early side effects. Three to four months after HT 3 patients deceased and 6 patients were lost to follow up. Overall 18\% (3/17) showed local progression and 29\% (5/17) had systemic progression.

The median local recurrence free survival (LRFS) after radiotherapy was 21 months for the primary treatment group and 10 months for the recurrence group. Median overall survival (OS) after HT was 57 months (0-120 months) for the primary treatment group and 11 months (0.3-22 months) for the recurrence group. In the primary treatment group four patients died of systemic progress within the observing interval. In the recurrence group seven patients died of systemic progress within the observing interval. Median progression free survival (PFS) after HT was 43 months for the primary treatment group and 7 months for the recurrence group. Figure 2 depicts the overall survival (OS), progression free survival (PFS) and local recurrence free survival (LRFS) after HT for each group. 
Table 2 Treatment Parameters

\begin{tabular}{|c|c|c|}
\hline & Recurrence Treatment Group $(n=12)$ & Primary Treatment Group $(n=14)$ \\
\hline Concurrent chemotherapy & $\begin{array}{l}3 \text { patients vinorelbin } \\
4 \text { patients capecitabin } \\
1 \text { patient sunitinib } \\
1 \text { patient doxorubicin }\end{array}$ & $\begin{array}{l}1 \text { patient vinorelbin } \\
1 \text { patient capecitabin }\end{array}$ \\
\hline \multicolumn{3}{|l|}{ PTV localization/extension ${ }^{a}$} \\
\hline a) Ipsilateral chest wall & 2 patients & 2patients \\
\hline $\begin{array}{l}\text { b) More than } 1 / 2 \text { of the thoracic circumference } \\
\text { plus loco-regional lymph nodes }\end{array}$ & 4 patients & 9 patients \\
\hline $\begin{array}{l}\text { c) More than } 2 / 3 \text { of the thoracic circumference } \\
\text { plus loco-regional lymph nodes }\end{array}$ & 6 patients & 3 patients \\
\hline d) Extranodal metastasis within the PTV & 1 patient (pleura) & 2 patients (bone lesions) \\
\hline e) Extensive skin metastases & 4 patients & \\
\hline PTV volume $\left(\mathrm{cm}^{3}\right)$ & $2984(1457-6837)$ & $1330(520-6623)$ \\
\hline Cranio-caudal PTV extension (cm) & $30(19-52)$ & $28(18,2-43,5)$ \\
\hline Median total dose & 40 Gy (32-60 Gy) & 50 Gy (40-60 Gy) \\
\hline Median dose per fraction & 2 Gy (1,8-3,0 Gy) & 2,0 Gy (1,5-2,24 Gy) \\
\hline Simultaneous integrated boost & $\begin{array}{l}2 \text { patients }(50.4 / 56 \text { Gy with single } \\
\text { doses of } 1.8 / 2.0 \mathrm{~Gy} \text {; } \\
55,8 / 60,1 \mathrm{~Gy} \text { with single doses } \\
\text { of } 1.8 / 1.94 \mathrm{~Gy} \text { ) }\end{array}$ & $\begin{array}{l}3 \text { patients ( } 45 / 50 \mathrm{~Gy} \text { with single doses } \\
\text { of } 1.8 / 2.0 \mathrm{~Gy} \text {; } \\
45 / 50 / 56 \mathrm{~Gy} \text { with single doses } \\
\text { of } 1.8 / 2.0 / 2.24 \mathrm{~Gy} \text {; } \\
46 \mathrm{~Gy} / 50 \text { with single doses of } 2.0 / 2.17 \mathrm{~Gy} \text { ) }\end{array}$ \\
\hline Neutron boost & 6 patients (4.6-12Gy) & - \\
\hline
\end{tabular}

${ }^{a}$ The PTV comprised more than a hemi-thorax in 13 and more than two thirds of the thoracic circumference in 9 patients

\section{Discussion}

Patients with locally advanced breast cancer in the primary setting are managed in a multidisciplinary approach. Operable tumors may be managed with modified radical mastectomy followed by chemotherapy

Table 3 Acute side effects during treatment with HT and concurrent chemotherapy

\begin{tabular}{llll}
\hline $\begin{array}{lll}\text { Common Toxicity } \\
\text { Criteria (CTC) }\end{array}$ & $\begin{array}{l}\text { All } \\
\text { patients }\end{array}$ & $\begin{array}{l}\text { Recurrence } \\
\text { Treatment Group }\end{array}$ & $\begin{array}{l}\text { Primary } \\
\text { Treatment Group }\end{array}$ \\
\hline Radiotherapy & $n=26$ & $n=12$ & $n=14$ \\
Radiodermatitis $^{\circ} 1$ & $14(53 \%)$ & $6(50 \%)$ & $8(57 \%)$ \\
Radiodermatitis $^{\circ} 2$ & $6(23 \%)$ & $4(33 \%)$ & $2(14 \%)$ \\
Radiodermatitis $^{\circ} 3$ & $6(23 \%)$ & $2(16.7 \%)$ & $4(28 \%)$ \\
Dysphagia $^{\circ} 1$ & $10(\%)$ & $5(42 \%)$ & $5(36 \%)$ \\
Lymphedema $^{\circ} 3$ & $3(11 \%)$ & $4(33 \%)$ & $1(7 \%)$ \\
Fatigue & $12(46 \%)$ & $5(42 \%)$ & $7(50 \%)$ \\
Chemotherapy & $n=11$ & $n=9$ & $n=2$ \\
Leucopenia ${ }^{\circ} 1$ & $6(55 \%)$ & $5(55 \%)$ & $1(50 \%)$ \\
Leucopenia 2 & $5(42 \%)$ & $4(44 \%)$ & $1(50 \%)$ \\
Anemia 1 & $6(55 \%)$ & $6(67 \%)$ & - \\
Anemia 2 & $3(27 \%)$ & $2(22 \%)$ & $1(50 \%)$ \\
Anemia 3 & $1(9 \%)$ & - & $1(50 \%)$ \\
Hand-foot- & $2(18 \%)$ & $2(22 \%)$ & - \\
syndrome & & & \\
\hline
\end{tabular}

and locoregional radiotherapy. Inoperable tumors may be treated with neoadjuvant chemotherapy followed by operation and radiotherapy. Some departments perform combined treatment with hyperthermia. Postoperative radiotherapy (chest wall and regional lymphatics) decreases the risk of local recurrence after mastectomy and improves overall survival for patients with high risk of local recurrence [11-13]. Internal mammary and supraclavicular irradiation for breast cancer reduces breast-cancer mortality and improves disease-free survival and distant disease-free survival [14].

For patients whose tumors remain inoperable after neoadjuvant chemotherapy the management is less clear. Some studies have used radiochemotherapy or radiation alone performed with conventional radiation techniques as an attempt to downsize the tumor mass before surgery [15-17].

It is still unclear what is the best technique for treating extensive disease in breast cancer patients. Some plan comparison studies are available on this topic [3, 4, 18-21]. Zhang et al. evaluated the dosimetric benefit of volumetric modulated arc therapy (VMAT) on postmastectomy left-sided breast cancer patients, with the involvement of internal mammary nodes [21]. VMAT achieves similar or superior target coverage and a better normal tissue sparing as compared to intensitymodulated radiotherapy (IMRT). Haciislamoglu et al. evaluated the dose distribution and homogeneity of 
Table 4 Local outcome and palliative effects

\begin{tabular}{|c|c|c|c|}
\hline & All patients & Recurrence Treatment Group & Primary Treatment Group \\
\hline End of $\mathrm{HT}$ & $n=26$ & $n=12$ & $n=14$ \\
\hline Local partial remission & $6(23 \%)$ & $4(33 \%)$ & $2(14 \%)$ \\
\hline Local complete remission & - & - & - \\
\hline Local stable disease & $20(76 \%)$ & $6(50 \%)$ & $12(85 \%)$ \\
\hline Local progression & $2(8 \%)$ & $2(17 \%)$ & 0 \\
\hline Systemic progression & $4(15 \%)$ & $2(17 \%)$ & $2(14 \%)$ \\
\hline Acute symptom relief & $7(27 \%)$ & $5(42 \%)$ & $2(14 \%)$ \\
\hline 6-8 weeks after HT & $n=23$ & $n=12$ & $n=11$ \\
\hline Local partial remission & $8(35 \%)$ & $6(50 \%)$ & $2(18 \%)$ \\
\hline Local complete remission & $4(18 \%)$ & $4(33 \%)$ & - \\
\hline Local stable disease & $7(30 \%)$ & - & $7(64 \%)$ \\
\hline Local progression & $1(4 \%)$ & - & $1(9 \%)$ \\
\hline Systemic progression & $3(13 \%$ & $2(17 \%)$ & $1(9 \%)$ \\
\hline Lost to follow up & 2 & - & 2 \\
\hline Deceased & 1 & - & 1 \\
\hline 3-4 months after HT & $n=17$ & $n=8$ & $n=9$ \\
\hline Local partial remission & $2(11 \%)$ & - & $2(22 \%)$ \\
\hline Local complete remission & - & - & - \\
\hline Local stable disease & $7(42 \%)$ & $3(38 \%)$ & $4(44 \%)$ \\
\hline Local progression & $3(18 \%)$ & $1(13 \%)$ & $2(22 \%)$ \\
\hline Systemic progression & $5(29 \%)$ & $4(50 \%)$ & $1(11 \%)$ \\
\hline Lost to follow up & 6 & 2 & 4 \\
\hline Deceased & 3 & 2 & 1 \\
\hline
\end{tabular}

four different types of IMRT (forward-planned IMRT, inverse-planned IMRT, HT and VMAT) in comparison with standard wedged tangential-beam 3D-CRT of the left breast in patients who had undergone lumpectomy [20]. All evaluated modalities provided adequate coverage of the whole breast. HT resulted in the lowest maximum doses delivered to the ipsilateral organs. Thus, if available, HT seems to be a good alternative. Chira et al. used neoadjuvant radiochemotherapy by helical tomotherapy for a group of 5 patients with locally advanced breast cancer [8]. All patients underwent mastectomy after radiotherapy. The preliminary results of Chira et al. showed that HT is feasible for neoadjuvant radiotherapy with acceptable toxicity profiles. Similarly, our patients treated in a primary setting with internal mammary and medial paraclavicular irradiation had low acute toxicities despite the very large treated volumes (maximum $6623 \mathrm{~cm}^{3}$ ). The median LRFS was 21 months and the median OS was 57 months.

Literature on reirradiation for locally advanced breast cancer recurrences with extensive skin infiltration and large bulky disease is sparse and not available on a systematic base concerning radiotherapy techniques or concurrent chemotherapy treatment. Some authors showed that reirradiation by 3D-CRT for patients with isolated local breast cancer recurrence should be considered as a salvage treatment with low to moderate toxicity and durable loco-regional control [7, 22-24]. Chatterjee et al. used HT for re-irradiation of three cases of supraclavicular disease from breast cancer. HT achieved a low dose to the brachial plexus without symptoms of brachial plexopathy and good local control of the supraclavicular disease [25]. Würschmidt et al. treated a heterogeneous group of 29 patients with reirradiation of locoregional recurrences of breast cancer [24]. They analyzed patients with operable (R1 or R0 resection) and non-operable recurrent breast tumors with and without distant metastases. However, most patients treated in this study had only a (extensive) thoracic wall recurrence, with just some patients with the axilla as simultaneous recurrence site. Thus the different outcome as compared to our data might be due to a more extensive disease treated in our cohort (for eg only two of our patients' had a chest wall reirradiation only). In our group of patients reirradiation was given with a median dose of $40 \mathrm{~Gy}$ to 


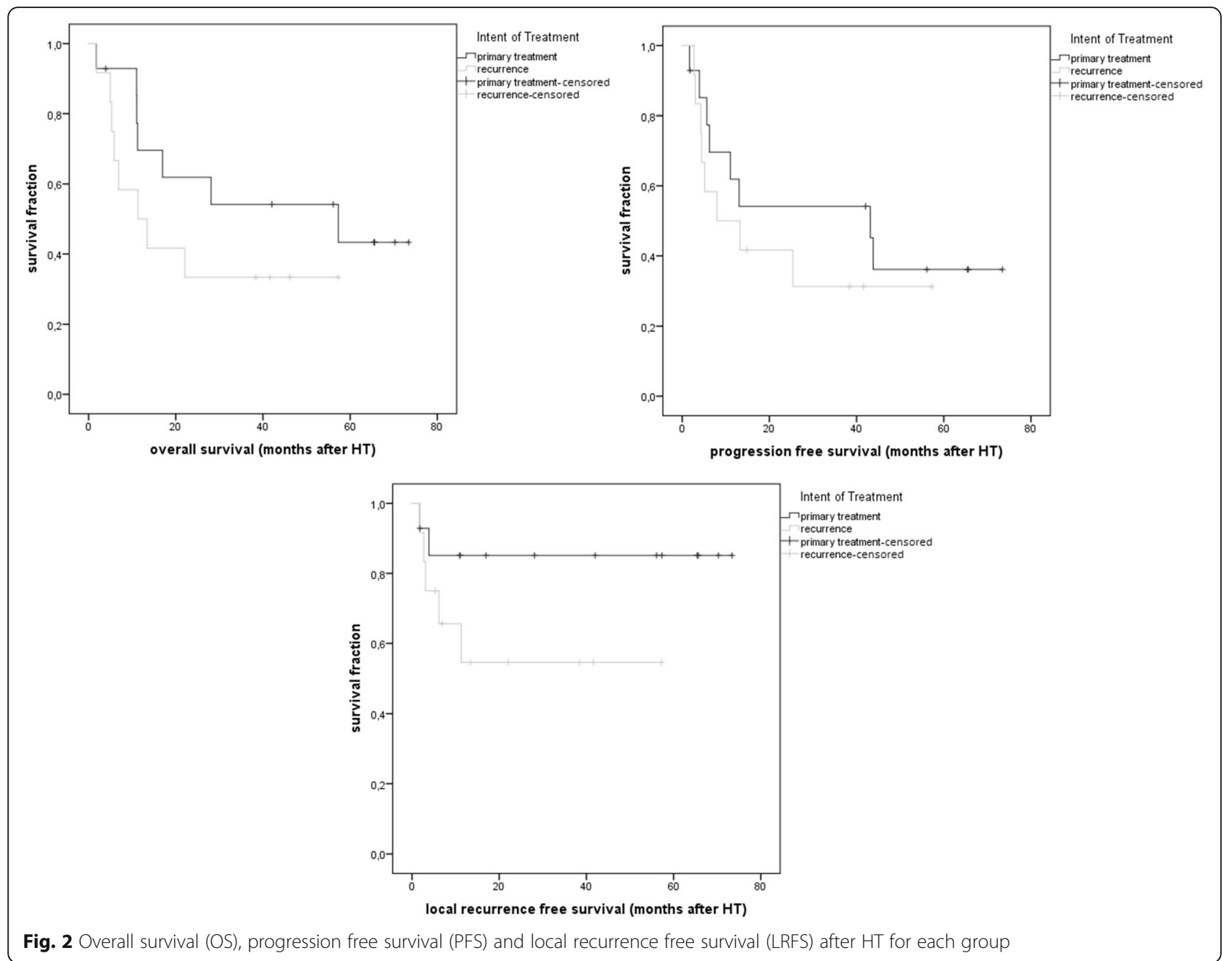

the PTV. Reirradiation was combined with concomitant chemotherapy in nine cases. In our experience concurrent chemotherapy with reirradiation is feasible in breast cancer without higher acute toxicity. We chose mostly capecitabine as concurrent chemotherapy regimen because of the high clinical experience in combination with radiotherapy treatment.

There are no recommendations regarding DVH data and normal tissue complication probabilities when very large breast tumors are treated with IMRT, VMAT or tomotherapy. For most of our analyzed patients, we recommended constraints based on Emami et al. [9]. Few patients were treated after 2010 with constraints based on QUANTEC [26]. The V20 and V30 are within the known constraints; however the V5 in our patients is fairly high as compared to the up to date recommendations. These deviations from internal and external standard operation procedures (SOP) is predominantly due to patients' anatomy as well as complexity of the target volumes, and were one of the indications why tomotherapy was chosen to treat the patients. Generally, dose constraints as well as accepted tolerances are individual decisions made for every single patient, and weighing of target coverage, applied dose and sparing of normal tissue has to be done thoroughly. Due to the retrospective nature of the study, no prospective long-term follow-up data is available; currently, long-term observations are in process to assess the long-term toxicites in these patients. One recent publication assessed nine breast cancer patients with a mean followup of 10.3 months [27]. A longer follow-up was available in 6 patients and the authors reported a pneumonitis in one of the 6 patients. This patient had a V20 of $25 \%$ and V5 of $64 \%$ and a preexisting lung disease. Nonetheless, the lung V20 for all patients ranged from 25 to 35\% (mean 29\%) and the V5 for all patients ranged from 51 to $75 \%$ (mean 66\%); thus an exclusively dosimetric explanation could not be offered by the authors [27]. Further studies are needed on this topic. 


\section{Conclusion}

HT is feasible for locally advanced and recurrent thorax embracing breast cancers with acceptable acute toxicity and a good therapy effect. Depending on the volume size, complexity as well as the individual patient's anatomy, the choice of helical IMRT has to be made and can lead to improved treatments plans with convincing outcome.

\section{Acknowledgements}

Not applicable.

\section{Funding}

None.

\section{Availability of data and materials}

The datasets supporting the conclusions of this article are included within the article.

\section{Authors' contributions}

MND, CH, CS, BC, MD and SK participated in the study design, contributed to the data collection, and draft the manuscript. SEC made important contributions in revising the content. MM contributed to data collection and analysis. All authors read and approved the final manuscript.

\section{Competing interests}

The authors declare that they have no competing interests.

\section{Consent for publication}

Not applicable.

\section{Ethics approval and consent to participate}

The study was evaluated by the ethics committee of Klinikum rechts der Isar. Consent to publish was obtained.

\section{Author details}

${ }^{1}$ Department of Radiation Oncology, Klinikum rechts der Isar der Technischen Universität München, Ismaninger Str. 22, 81675 München, Germany. ${ }^{2}$ Praxis für Strahlentherapie, Hausham, Germany. ${ }^{3}$ Institute of Innovative Radiotherapy (iRT), Helmholtz Zentrum München, München, Germany.

Received: 19 February 2016 Accepted: 1 December 2016

Published online: 28 January 2017

\section{References}

1. Jobsen JJ, van der Palen J, Meerwaldt JH. The impact of age on local control in women with $\mathrm{pT} 1$ breast cancer treated with conservative surgery and radiation therapy. Eur J Cancer. 2001:37(15):1820-7.

2. Aberizk WJ, et al. The use of radiotherapy for treatment of isolated locoregional recurrence of breast carcinoma after mastectomy. Cancer. 1986;58(6):1214-8.

3. Goddu SM, et al. Helical tomotherapy planning for left-sided breast cancer patients with positive lymph nodes: comparison to conventional multiport breast technique. Int J Radiat Oncol Biol Phys. 2009;73(4):1243-51.

4. Caudrelier JM, et al. Helical tomotherapy for locoregional irradiation including the internal mammary chain in left-sided breast cancer: dosimetric evaluation. Radiother Oncol. 2009:90(1):99-105.

5. Kainz K, et al. Investigation of helical tomotherapy for partial-breast irradiation of prone-positioned patients. Int J Radiat Oncol Biol Phys. 2009;74(1):275-82.

6. Gonzalez VJ, et al. Evaluation of two tomotherapy-based techniques for the delivery of whole-breast intensity-modulated radiation therapy. Int J Radiat Oncol Biol Phys. 2006;65(1):284-90.

7. Harkenrider MM, Wilson MR, Dragun AE. Reirradiation as a component of the multidisciplinary management of locally recurrent breast cancer. Clin Breast Cancer. 2011;11(3):171-6.

8. Chira C, et al. Helical tomotherapy for inoperable breast cancer: a new promising tool. Biomed Res Int. 2013;2013:264306.

9. Emami B, et al. Tolerance of normal tissue to therapeutic irradiation. Int J Radiat Oncol Biol Phys. 1991;21(1):109-22.
10. Nieder $C$, et al. Update of human spinal cord reirradiation tolerance based on additional data from 38 patients. Int J Radiat Oncol Biol Phys. 2006;66(5):1446-9.

11. Sautter-Bihl ML, et al. DEGRO practical guidelines: radiotherapy of breast cancer III-radiotherapy of the lymphatic pathways. Strahlenther Onkol. 2014;190(4):342-51.

12. Wenz $F$, et al. DEGRO practical guidelines for radiotherapy of breast cancer IV: radiotherapy following mastectomy for invasive breast cancer. Strahlenther Onkol. 2014;190(8):705-14.

13. Ebctcg, et al. Effect of radiotherapy after mastectomy and axillary surgery on 10-year recurrence and 20-year breast cancer mortality: meta-analysis of individual patient data for 8135 women in 22 randomised trials. Lancet. 2014;383(9935):2127-35.

14. Poortmans PM, et al. Internal mammary and medial supraclavicular irradiation in breast cancer. N Engl J Med. 2015;373(4):317-27.

15. Bates $\mathrm{T}$, et al. Primary chemo-radiotherapy in the treatment of locally advanced and inflammatory breast cancer. Breast. 2012;21(3):330-5.

16. Matuschek $\mathrm{C}$, et al. Long-term outcome after neoadjuvant radiochemotherapy in locally advanced noninflammatory breast cancer and predictive factors for a pathologic complete remission : results of a multivariate analysis. Strahlenther Onkol. 2012;188(9):777-81.

17. Lerouge $\mathrm{D}$, et al. Combined chemotherapy and preoperative irradiation for locally advanced noninflammatory breast cancer: updated results in a series of 120 patients. Int J Radiat Oncol Biol Phys. 2004;59(4):1062-73.

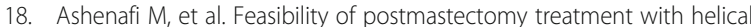
TomoTherapy. Int J Radiat Oncol Biol Phys. 2010;77(3):836-42.

19. Cendales $\mathrm{R}$, et al. Helical tomotherapy in patients with breast cancer and complex treatment volumes. Clin Transl Oncol. 2011;13(4):268-74.

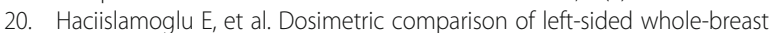
irradiation with 3DCRT, forward-planned IMRT, inverse-planned IMRT, helical tomotherapy, and volumetric arc therapy. Phys Med. 2015;31(4):360-7.

21. Zhang Q, et al. Dosimetric comparison for volumetric modulated arc therapy and intensity-modulated radiotherapy on the left-sided chest wall and internal mammary nodes irradiation in treating post-mastectomy breast cancer. Radiol Oncol. 2015;49(1):91-8

22. Harms $W$, et al. Results of chest wall reirradiation using pulsed-dose-rate (PDR) brachytherapy molds for breast cancer local recurrences. Int J Radiat Oncol Biol Phys. 2001:49(1):205-10.

23. Deutsch M. Repeat high-dose external beam irradiation for in-breast tumor recurrence after previous lumpectomy and whole breast irradiation. Int J Radiat Oncol Biol Phys. 2002;53(3):687-91.

24. Wurschmidt F, et al. Reirradiation of recurrent breast cancer with and without concurrent chemotherapy. Radiat Oncol. 2008;3:28.

25. Chatterjee $\mathrm{S}$, et al. Managing supraclavicular disease from breast cancer with brachial plexus-sparing techniques using helical tomotherapy. Clin Oncol (R Coll Radiol). 2011;23(2):101-7.

26. Bentzen SM, et al. Quantitative Analyses of Normal Tissue Effects in the Clinic (QUANTEC): an introduction to the scientific issues. Int J Radiat Oncol Biol Phys. 2010;76(3 Suppl):S3-9.

27. Kaidar-Person O, et al. Helical tomotherapy for bilateral breast cancer: Clinical experience. Breast. 2016;28:p79-83. 